\title{
Laparoscopic Treatment of Cesarean Scar Ectopic Pregnancy
}

\author{
Robert Hudeček, MD, PhD, Zuzana Felsingerová, MD, Michal Felsinger, MD, and Eva Jandakova, MD²
}

\begin{abstract}
Background: An ectopic pregnancy within a Cesarean scar represents a rare type of extrauterine pregnancy in which the fertilized egg nidates in the myometrium of the uterine wall within a scar left from a previous Cesarean delivery. An unrecognized growing Cesarian scar pregnancy may result in uterine rupture, uncontrollable metrorrhagia, and bleeding into the abdominal cavity; therefore, early diagnosis and therapy are necessary to prevent the development of severe complications. Case: A 34-year-old woman after a previous Cesarean delivery presented with amenorrhoa of 7 weeks' duration. Transvaginal ultrasonography revealed an ectopic pregnancy in the Cesarean scar, and a laparoscopic removal of the gestational sac was performed with no complications. Results: Three months later, another laparoscopy with chromopertubation showed no signs of penetration in the suture, both the Fallopian tubes being bilaterally passable. The patient was advised that she could try to achieve pregnancy through spontaneous conception, after which monitoring of the gestational development and a careful assessment of the nidation site would be needed. Conclusions: Laparoscopic surgical management of a viable ectopic pregnancy is technically simple, and is followed by a good recovery. ( $\mathrm{J}$
\end{abstract} GYNECOL SURG 30:309)

\section{Introduction}

A N ECTOPIC PREGNANCY WITHIN A CESAREAN SCAR (Cesarean scar pregnancy [CSP]) represents a rare type of extrauterine pregnancy in which the fertilized egg nidates in the myometrium of the uterine wall within a scar left from a previous Cesarean delivery (CD). An unrecognized growing CSP may result in uterine rupture, uncontrollable metrorrhagia, and bleeding into the abdominal cavity; therefore, early diagnosis and therapy are necessary to prevent the development of severe complications. ${ }^{1}$ The ultimate goal of the therapeutic procedure is to preserve the patient's fertility. CSP therapy includes conservative pharmacologic measures such as systemic application of methotrexate (MTX) or ultrasoundguided local application of hyperosmolar solutions. ${ }^{2}$ Further therapeutic options include conservative fertility-preserving types of surgery, such as selective uterine artery embolization; bilateral hypogastric artery ligation associated with trophoblastic evacuation; 3,4 dilatation and curettage; and a hysteroscopic, laparoscopic, or laparotomic removal of the CSP. ${ }^{5,6} \mathrm{~A}$ radical surgical approach would be the complete removal of the uterus, leading to definitive infertility.

\section{Case}

A 34-year-old woman, with a history of uncomplicated CD for intrapartum fetal hypoxia 4 years previously, presented in her second pregnancy with amenorrhoea of 7 weeks' duration. An ultrasound examination raised a suspicion of a Cesarean scar ectopic pregnancy. The patient had an enlarged and tender uterus, otherwise she was asymptomatic. On vaginal ultrasound examination, a viable singleton pregnancy $6+5$ (6 weeks and 5 days, based on crown-rump length [CRL]) located in the anterior wall of the uterus at the site of the Cesarean scar was found, protruding toward and under the vesical fold (Fig. 1). There was no free fluid in the rectouterine pouch, and admission laboratory test results were normal, with a serum human chorionic gonadotrophin (hCG) level of $7866 \mathrm{U} / \mathrm{L}$. A diagnostic laparoscopy confirmed the protrusion into the anterior wall of the uterus at the uterine isthmus, covered by the vesicouterine fold of the peritoneum (Fig. 2). A transcervical application of methylene blue showed discoloration in the protrusion.

A sharp dissection of the vesicouterine peritoneal fold and extirpation of the ectopic pregnancy located in the dehiscent scar was performed, followed by bipolar coagulation, cutting, and laparoscopic suturing of the defect in two layers followed by peritonealization (Fig. 3). Chromopertubation showed no signs of leakage in the uterine suture, and both the Fallopian tubes were open. A Redon drain was inserted into the rectouterine pouch and removed the following day. The patient was discharged on the 2 nd day following the

Departments of ${ }^{1}$ Obstetrics and Gynaecology, and ${ }^{2}$ Pathology, Masaryk University and University Hospital Brno, Brno, Czech Republic. 


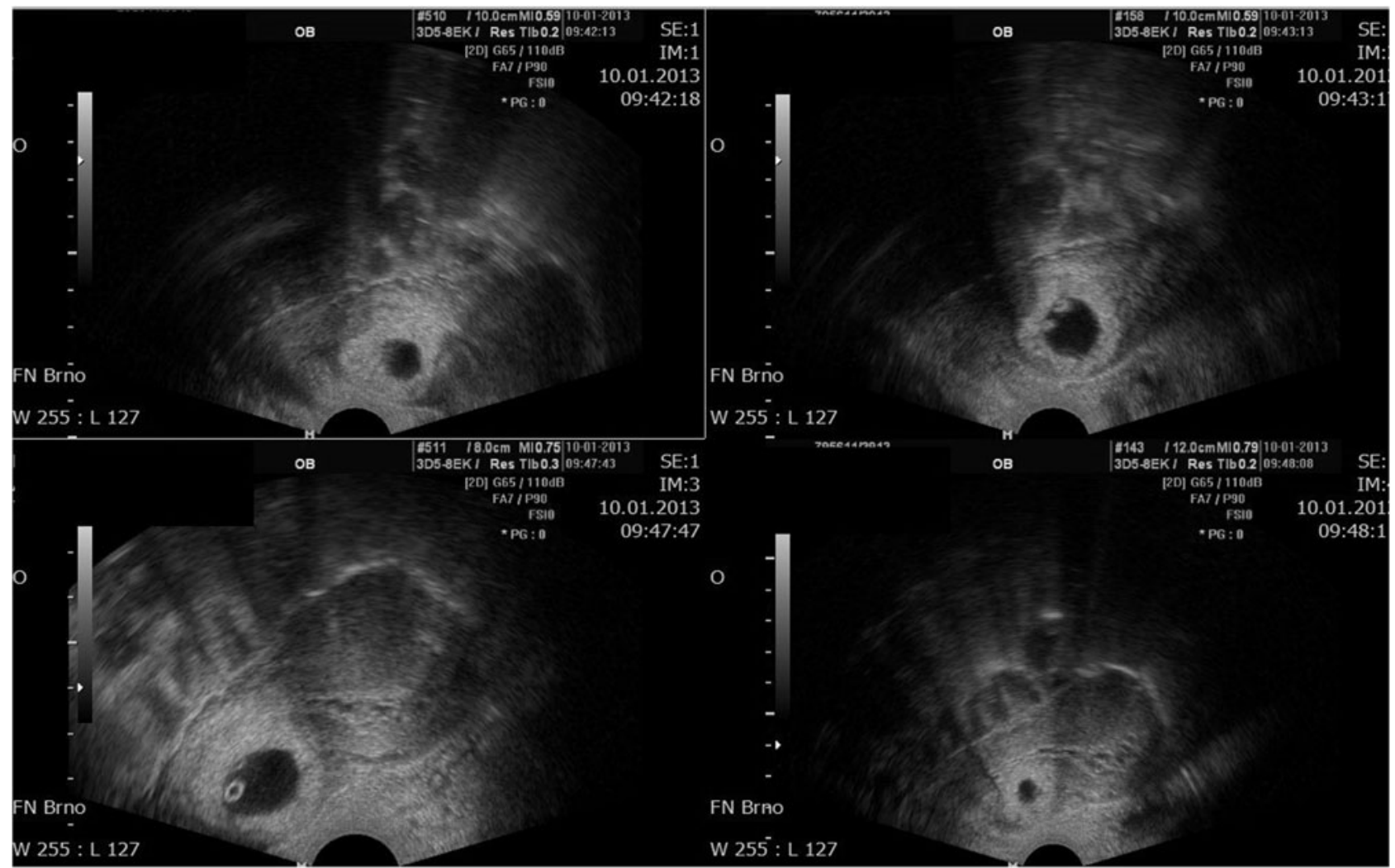

FIG. 1. Transvaginal ultrasound images of the Cesarean scar pregnancy with fetal cardiac activity.

intervention, and a histologic examination confirmed the existence of the ectopic pregnancy in the Cesarean scar tissue (Fig. 4). The follow-up was uneventful.

\section{Results}

Three months after the surgery, the patient underwent another laparoscopic inspection in which foci of endometriosis on the peritoneum and plica vesicouterina of the anterior wall of the uterus were detected. Chromopertuba-

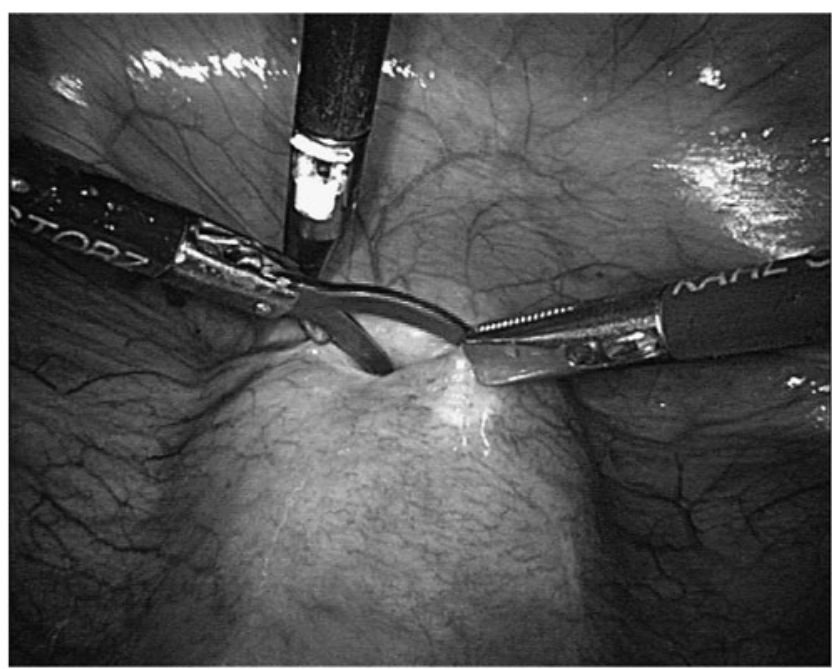

FIG. 2. Sharp dissection of the vesicouterine peritoneal fold, and visualization of the ectopic pregnancy in the $\mathrm{Ce}$ sarean section scar affected by dehiscence. tion did not reveal any leakage from the scar. The patient was advised that she could try to achieve pregnancy through spontaneous conception.

\section{Discussion}

In the present case study, it has been demonstrated that a viable CSP can be treated safely by conservative procedures, which include a laparoscopic removal of the pregnancy and a primary repair of the uterine defect. The removal of the ectopic

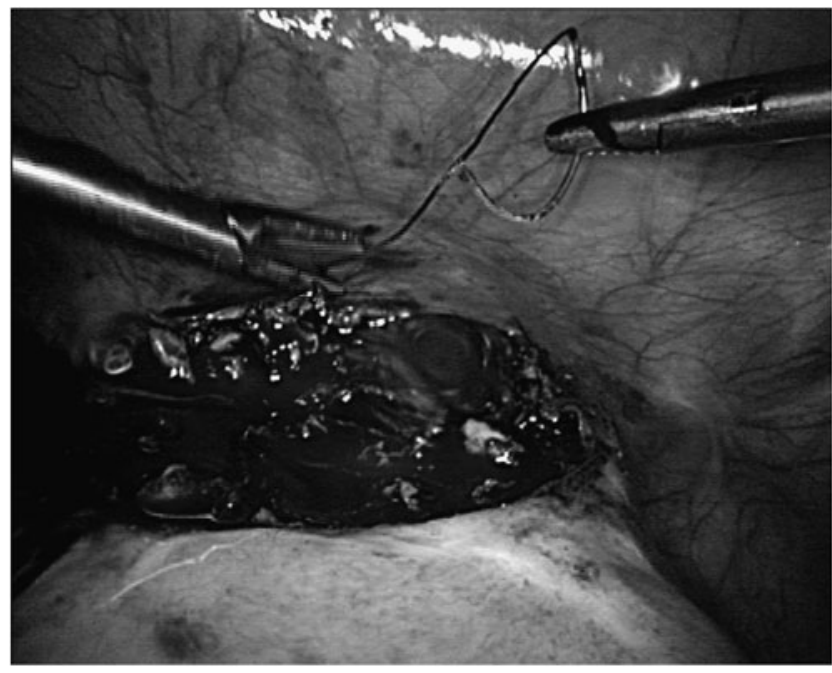

FIG. 3. Laparoscopic suture of the defect performed by stitches in two layers. 


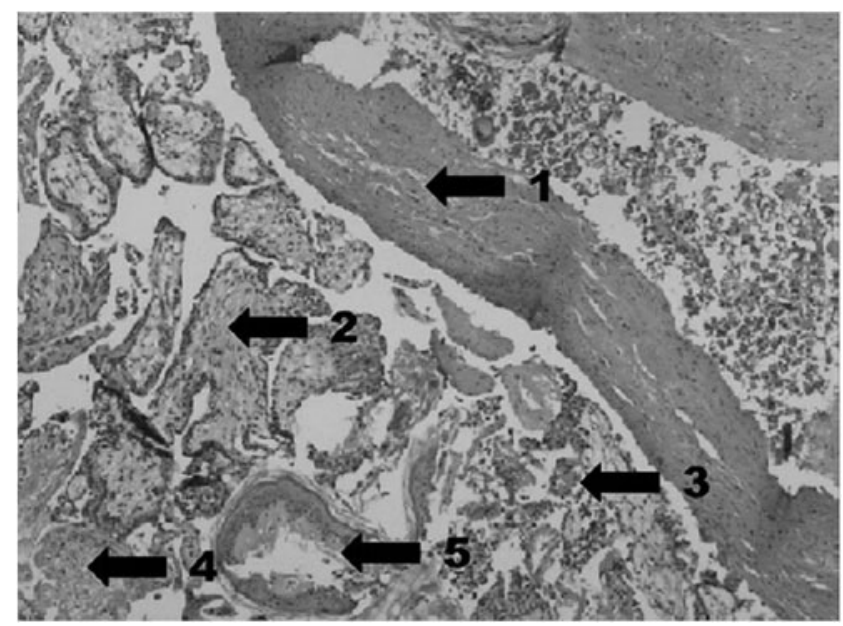

FIG. 4. Histologic description: 1, fibrin; 2, trophoblast; $\mathbf{3}$, syncytiotrophoblast; $\mathbf{4}$, chorionic villi; and $\mathbf{5}$, cellular debris.

gestational sac was technically simple, performed in a short time with minimal loss of blood, and followed by a good recovery. It is assumed that the blastocyst nidates in the endometrium or myometrium in an area with microscopic dehiscences within a CD scar. ${ }^{7}$ With increasing CD rates, an increased CSP incidence may be expected. Clinical symptoms may be few in the incipient phase of the pregnancy, but at later stages, a CSP may result in uterine rupture, accompanied by massive hemorrhage, hemoperitoneum and hemorrhagic shock.

Vaginal ultrasound with Doppler is the predominant diagnostic method used for CSP; this method also used to reveal location of implantation sites, to show discontinuity on the anterior uterine wall, and to show absent or diminished healthy myometrium between the bladder and the gestational sac. Because of the high velocity of the method combined with low impedance, the peritrophoblastic vascular flow clearly surrounding the sac may be seen. A protrusion in the isthmic part of the anterior uterine wall is diagnosed by laparoscopy.

To date, no optimal CSP therapy is known; current therapy usually involves pharmacologic, surgical, or, sometimes, combined approaches. The surgical approaches may be either radical (hysterectomy), or conservative (as in the present case). The pregnancy must be removed, with the subsequent repair of the uterine defect by laparotomy, laparoscopy, or hysteroscopy. ${ }^{8,9}$ A bilateral hypogastric artery ligation associated with the evacuation of the pregnancy and a repair of the uterine defect under laparoscopic guidance has been described as successful. ${ }^{10}$ How well the uterine scar left after the treatment heals, and what may occur in future pregnancies, is not known. Long-term outcomes to be considered are future fertility and a possible recurrence of CSP. Favorable reproductive outcomes and a low recurrence rate have been described. ${ }^{11}$ Treatment decisions depend on gestational age, hCG levels, the presence of fetal cardiac activity, the desire for future fertility, and the experience and facilities available. Counselling for patients with CSP is not easy, as not enough information is available so as to recommend the optimal treatment.

\section{Conclusions}

Laparoscopic surgical managment of a viable ectopic pregnancy is technically simple, and is followed by good recovery.

\section{Acknowledgments}

This work was supported by government grant MZ ČR RVO (FNBr, 65269705).

\section{Disclosure Statement}

No competing financial interests exist.

\section{References}

1. Herman A, Weinraub Z, Avrech O, Mamon R, Ron-El R, Bukovsky Y. Follow up and outcome of isthmic pregnancy located in a previous caesarean section scar. Br J Obstet Gynaecol 1995;102:839.

2. Persadie RJ, Fortier A, Stopla RG. Ectopic pregnancy in a caesarean scar: A case report. J Obstet Gynaecol Can 2005;27:1102.

3. Sugawara J, Senoo M, Chisaka H, Yaegashi N, Okanuta K. Successful conservative treatment of a Cesarean scar pregnancy with uterine artery embolization. Tohoku J Exp Med 2005;206:261.

4. Yang MJ, Jeng MH. Combination of transarterial embolization of uterine arteries and conservative surgical treatment for pregnancy in a Cesarean section scar: A report of 3 cases. J Reprod Med 2003;48:213.

5. Seow KM, Cheby WC, Chuang J, Lee C, Tsai YL, Hwang JL. Methotrexate for Cesarean scar pregnancy after in vitro fertilization and embryo transfer: A case report. J Reprod Med 2000;45:754.

6. Godin PA, Bassil S, Donnez J. An ectopic pregnancy developing in a previous caesarian section scar, Fertil Steril 1997;67:398.

7. Seow KM, Hwang JL, Tsai YL, Juany LW, Lin YH, Hsieh BC. Subsequent pregnancy outcome after conservative treatment of a previous cesarean scar pregnancy. Acta Obstet Gynecol Scand 2004;83:1167.

8. Deans R, Abbott J. Hysteroscopic management of cesarean scar ectopic pregnancy. Fertil Steril 2010;93:1735.

9. Hong SC, Lau M, Yam P. Ectopic pregnancy in previous Caesarean section scar Singapore Med J 2011;52: 115.

10. Kung FT, Juany TL, Chen CW, Cheby YF. Cesarean scar ectopic pregnancy. Fertil Steril 2006;85:1508.

11. Nagi JB, Helmy S, Ofili-Yebovi D, Yazbek J, Sawyer E, Jurkovi D. Reproductive outcomes of women with a previous history of Caesarean scar ectopic pregnancies. Hum Reprod 2007;22:2012.

Address correspondence to: Robert Hudeček, $M D, P h D$

Department of Obstetrics and Gynaecology Masaryk University and University Hospital Brno Obilní trh 11 Brno 62500 Czech Republic

E-mail:rhudecek@fnbrno.cz 